\title{
Rapid Formation and Rupture of Multiple Abdominal Pseudoaneurysms: A Life Threatening Case of Segmental Arterial Mediolysis
}

\author{
Ernest M Cheng, MD, ${ }^{1,2}$ Kerry L Chen, BMed, MD, ${ }^{1,2}$ Varsha Sharma, MBBS, FRACS, ${ }^{1}$ \\ Juliana Yee, MBBS, ${ }^{3}$ Mark Power, BAppSc, MBBS, FRANZCR, ${ }^{3}$ \\ Lubomry D Lemech, MBBS (Hons), ${ }^{4}$ and Francis Chu, MBBS, FRACS ${ }^{1,2}$
}

We present a 62-year-old gentleman with rapidly forming abdominal pseudoaneurysms due to segmental arterial mediolysis (SAM). With rupture of his pseudoaneurysms, he underwent angiography and successful coil embolisation. In this case, we demonstrate the potential for rapid progression of pseudoaneurysms in SAM, with the need for prompt diagnosis and urgent endovascular intervention.

Keywords: segmental arterial mediolysis, coil embolization, pseudoaneurysms

\section{Introduction}

Segmental arterial mediolysis (SAM) is an uncommon but critical condition with up to a $60 \%$ mortality rate in the acute phase from catastrophic hemorrhage. It is a non-inflammatory, non-atherosclerotic vasculopathy with segmental lysis of the tunica medial layer of abdominal

\footnotetext{
${ }^{1}$ Department of Surgery, St George Hospital, Sydney, Australia

${ }^{2}$ St George Clinical School, University of New South Wales, Sydney, Australia

${ }^{3}$ Department of Radiology, St George Hospital, Sydney, Australia

${ }^{4}$ Department of Vascular Surgery, St George Hospital, Sydney, Australia
}

Received: December 20, 2020; Accepted: May 31, 2021 Corresponding author: Ernest M Cheng, MD. Department of Surgery, St George Hospital, Sydney NSW 2217, Australia Tel: +61-435115676, Fax: +61-291134887

E-mail: ernestmcheng@gmail.com

(cc) BY-NC-SA (92021 The Editorial Committee of Annals of Vascular Diseases. This article is distributed under the terms of the Creative Commons Attribution License, which permits use, distribution, and reproduction in any medium, provided the credit of the original work, a link to the license, and indication of any change are properly given, and the original work is not used for commercial purposes. Remixed or transformed contributions must be distributed under the same license as the original. arteries. ${ }^{1)}$ SAM most commonly affects coeliac, mesenteric and renal arteries, and its presentation varies from chronic mesenteric ischaemia to acute life-threatening haemorrhage thus posing both a diagnostic and therapeutic challenge. ${ }^{1,2}$ ) We report a case of SAM in a 62-year-old gentleman presenting with rapid formation and rupture of pseudoaneurysms who was successfully treated with early coil embolisation.

\section{Case Report}

A 62-year old gentleman presented with sudden onset, severe generalised abdominal pain. He had normal vitals signs but on examination demonstrated generalised peritonism. Biochemistry showed lactataemia of $4.2 \mathrm{mmol} / \mathrm{L}$ but was otherwise unremarkable. Computer tomography (CT) imaging of his abdomen was equivocal for an early superior mesenteric artery dissection. Spontaneous and complete resolution of the patient's symptoms occurred within an hour of presentation and he was admitted for monitoring. Twenty-four hours later the patient experienced the same severe, sudden onset, abdominal pain accompanied by hypotension and syncope. A CT mesenteric angiogram demonstrated two pseudoaneurysms arising from the splenic artery with associated rupture of the proximal pseudoaneurysm and retroperitoneal haemorrhage (Fig. 1). The proximal pseudoaneurysm was embolised by interventional radiology. Four hours later, the patient became haemodynamically unstable with a significant haemoglobin drop $(24 \mathrm{~g} / \mathrm{L})$ requiring massive transfusion. Repeat CT mesenteric angiogram demonstrated new left gastric, short gastric and distal splenic pseudoaneurysms with rupture of the distal splenic pseudoaneurysm. Coil embolisation was performed on the haemorrhaging distal splenic artery pseudoaneurysm and preventatively on the left gastric artery pseudoaneurysm (Fig. 2). The patient had no further bleeds and was monitored in inten- 
Cheng EM, et al.
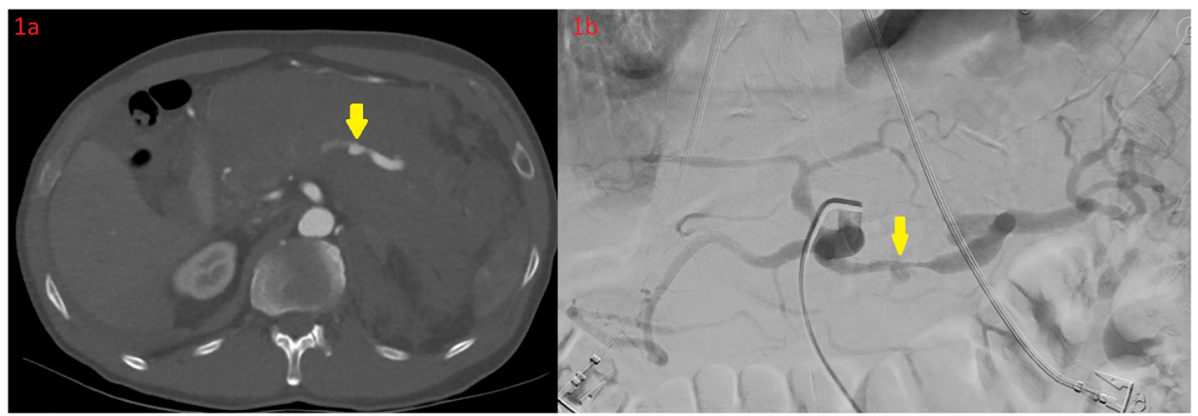

Fig. 1 (a) Computed tomography angiogram demonstrating splenic artery pseudoaneurysm surrounded by retroperitoneal haemorrhage. (b) Angiography demonstrating proximal splenic artery pseudoaneurysm.

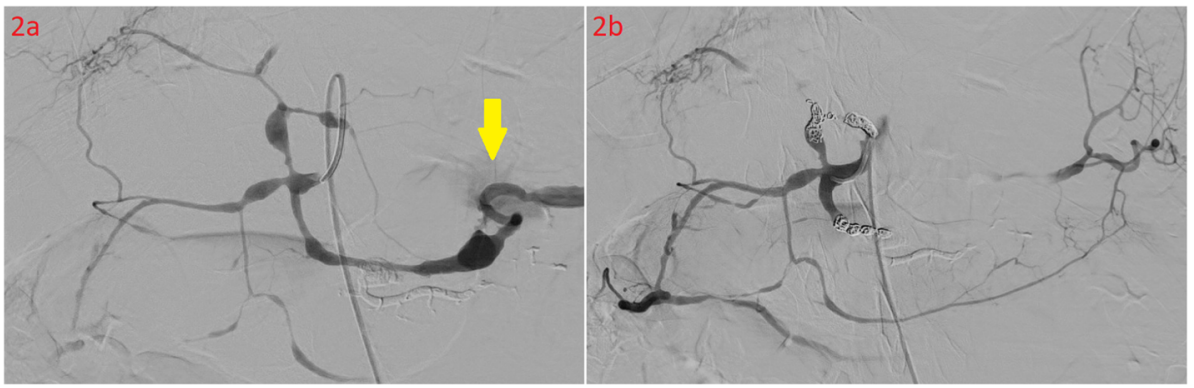

Fig. 2 (a) Before embolization: active bleeding from the distal splenic pseudoaneurysm, new left gastric artery pseudoaneurysm and previous embolization site of proximal splenic artery. (b) Post-embolisation of left gastric artery and distal splenic pseudoaneurysms.

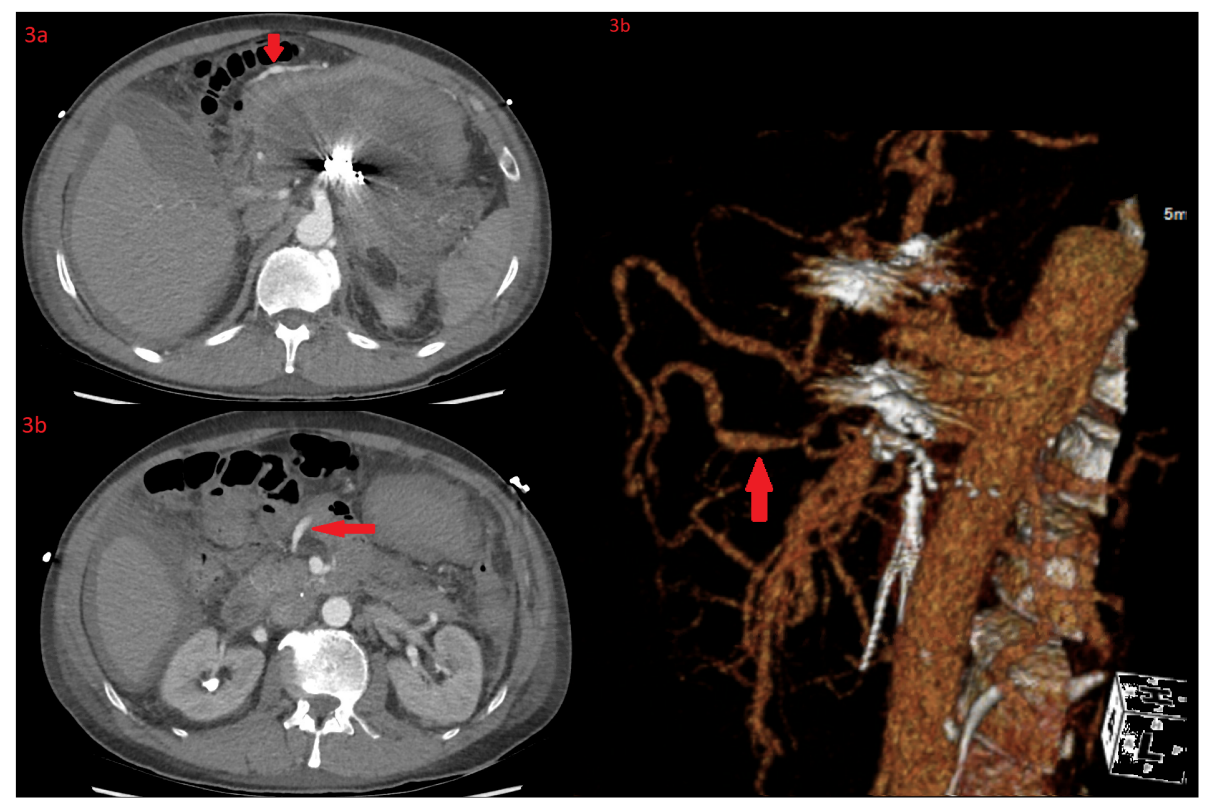

Fig. 3 (a) "String of beads" radiographic findings seen at the gastroepiploic artery. (b) Mid superior mesenteric artery dilatation on computed tomography. (c) 3-Dimensional reconstruction of the mid superior mesenteric artery dilatation.

sive care unit. Subsequent CT angiogram demonstrated further aneurysmal dilatations along the gastroepiploic artery, middle colic branch of the superior mesenteric artery (SMA) and left common iliac artery (Fig. 3). A dissection flap at the mid SMA was also noted, however, this was present on previous CT imaging and unchanged. All 
vasculitis, thrombophilic and infective screens were negative. This patient's clinical presentation combined with the radiological findings were consistent with SAM.

His recovery was complicated by multiple segmental and subsegmental pulmonary embolisms requiring insertion of an inferior vena cava filter and therapeutic anticoagulation. However, he had no further haemorrhagic events. He presented 3 months later after falling off a horse and suffered 6 rib fractures on his left side. Despite the significant force from the fall, abdominal imaging demonstrated stable aneurysmal dilatations and the patient demonstrated no further abdominal bleeding episodes. He was followed up 6 months after the event with no further bleeding.

\section{Discussion}

SAM is a rare vascular disorder with unknown aetiology first described by Slavin and Gonzalez-Vitale in 1976.1) It characteristically affects medium to large gastrointestinal arteries and causes degeneration of the tunica media without inflammatory, atherosclerotic or hereditary components. ${ }^{3)}$ Although there are no proven risk factors, hypertension is commonly associated with SAM in the literature..$^{2)}$ There are currently no known precipitating factors for an acute episode of the disease and patients typically present with sudden abdominal pain secondary to dissection, ischaemia or haemorrhage. ${ }^{2,4)}$ SAM poses a diagnostic challenge as clinical presentations can vary from a brief episode of abdominal pain with stable disease to significant haemodynamic instability from haemorrhage. As such, the natural history of SAM is poorly understood and the estimated mortality rate of SAM varies from 0 to $60 \% .{ }^{2,5,6)}$ Diagnosis of SAM may not require histopathological confirmation, as CT findings of arterial dissection, fusiform aneurysms, wall thickening and "string of beads" appearance affecting abdominal visceral arteries are often sufficient for diagnosis. ${ }^{2)}$ Kalva et al. described a noninvasive diagnostic criteria for SAM. Its criterion is based on clinical and radiological findings described above and the absence of congenital predisposition for dissection (e.g. Ehlers-Danlos, Marfan, Leoys-Dietz) and a low suspicion of alternative arteriopathies (e.g. fibromuscular dysplasia, collagen vascular disorders or arteritis). Negative inflammatory markers such as antinuclear antibodies and antineutrophil cytoplasmic antibodies contributed to the diagnosis of SAM.7) However, tissue diagnosis, if obtainable, can assist in differentiating mimics of SAM include anti-neutrophil cytoplasmic autoantibody-associated vasculitis, polyarteritis nodosa, C and mycotic aneurysmal infections. ${ }^{8}$ In cases where the early phase of SAM when the diagnosis is unclear, a combination of tissue diagnosis, vasculitis biomarkers and infection screens can be useful in distinguishing similar arteriopathies. ${ }^{2)}$

Treatment for SAM in the acute setting includes emergent endovascular intervention, open surgery or medical management. ${ }^{2,5,8)}$ Shenouda et al. reviewed 85 cases of SAM with 24 cases utilising endovascular intervention and 36 cases undergoing emergency open laparotomy. Open surgery was associated with poorer outcomes with $9 \%$ mortality compared to $0 \%$ in those undergoing endovascular treatment. ${ }^{8)}$ However, open surgery is valuable in the event of failed endovascular intervention or in the elective setting for vessel ligation for known stable pseudoaneurysms. ${ }^{8,9)}$ Alternatively, patients who survived the acute phase of the disease can be managed medically with anti-hypertensives, antiplatelets or anticoagulation in combination with serial CT imaging. ${ }^{5)}$ Fortunately, disease regression and stability were observed in many patients after resolution of their initial acute episode. Several studies have concluded after the acute phase, majority of patients demonstrated regression or stability of disease..$^{5,10,11)}$

\section{Conclusion}

Vascular surgeons should be aware of SAM as a differential for patients presenting with acute abdominal pain. SAM as a life-threatening condition requiring the need for early angiography and embolization via interventional radiology, rather than diagnostic open exploratory laparotomy. This case contributes to our understanding of the variability in SAM's clinical presentation and knowledge of its potential to rapidly propagate pseudoaneurysms at risk of rupture. It emphasises the need for vigilance when approaching abdominal pain with initially unclear aetiology.

\section{Disclosure Statements}

Formal written consent was obtained from the patient for use of patient imaging.

The authors declare that there is no conflict of interest regarding the publication of this article.

\section{Author Contributions}

Study conception: EC, VS, MP, LL, FC

Data collection: EC, JY, MP

Analysis: EC, KC, VS, JY

Investigation: $\mathrm{EC}, \mathrm{KC}$

Writing: EC, KC, JY

Critical review and revision: all authors

Final approval of the article: all authors

Accountability for all aspects of the work: all authors 


\section{References}

1) Slavin RE, Inada K. Segmental arterial mediolysis with accompanying venous angiopathy: a clinical pathologic review, report of 3 new cases, and comments on the role of endothelin-1 in its pathogenesis. Int J Surg Pathol 2007; 15: 121-34.

2) Skeik N, Olson SL, Hari G, et al. Segmental arterial mediolysis (SAM): systematic review and analysis of 143 cases. Vasc Med 2019; 24: 549-63.

3) Chao CP. Segmental arterial mediolysis. Semin Intervent Radiol 2009; 26: 224-32.

4) Akuzawa N, Kurabayashi M, Suzuki T, et al. Spontaneous isolated dissection of the superior mesenteric artery and aneurysm formation resulting from segmental arterial mediolysis: a case report. Diagn Pathol 2017; 12: 74.

5) Naidu SG, Menias CO, Oklu R, et al. Segmental arterial mediolysis: abdominal imaging of and disease course in 111 patients. AJR Am J Roentgenol 2018; 210: 899-905.

6) Tameo MN, Dougherty MJ, Calligaro KD. Spontaneous dissection with rupture of the superior mesenteric artery from segmental arterial mediolysis. J Vasc Surg 2011; 53: 1107-12.

7) Kalva SP, Somarouthu B, Jaff MR, et al. Segmental arterial mediolysis: clinical and imaging features at presentation and during follow up. J Vasc Interv Radiol 2011; 22: 1380-7.

8) Shenouda M, Riga C, Naji Y, et al. Segmental arterial mediolysis: a systematic review of 85 cases. Ann Vasc Surg 2014; 28: 269-77.

9) Hashimoto T, Deguchi J, Endo H, et al. Successful treatment tailored to each splanchnic arterial lesion due to segmental arterial mediolysis (SAM): report of a case. J Vasc Surg 2008; 48: 1338-41.

10) Sakano T, Morita K, Imaki M, et al. Segmental arterial mediolysis studied by repeated angiography. $\mathrm{Br} \mathrm{J}$ Radiol 1997; 70: 656-8.

11) Michael M, Widmer U, Wildermuth $S$, et al. Segmental arterial mediolysis: CTA findings at presentation and followup. AJR Am J Roentgenol 2006; 187: 1463-9. 